\title{
ON SOME REAL HYPERSURFACES OF A COMPLEX PROJECTIVE SPACE
}

BY

\author{
MASAFUMI OKUMURA
}

\begin{abstract}
A principal circle bundle over a real hypersurface of a complex projective space $C P^{n}$ can be regarded as a hypersurface of an odddimensional sphere. From this standpoint we can establish a method to translate conditions imposed on a hypersurface of $C P^{n}$ into those imposed on a hypersurface of $s^{2 n+1}$. Some fundamental relations between the second fundamental tensor of a hypersurface of $C P^{n}$ and that of a hypersurface of $S^{2 n+1}$ are given.
\end{abstract}

Introduction. As is well known a sphere $S^{2 n+1}$ of dimension $2 n+1$ is a principal circle bundle over a complex projective space $C P^{n}$ and the Riemannian structure on $C P^{n}$ is given by the submersion $\pi: S^{2 n+1} \rightarrow C P^{n}$ [4], [7]. This suggests that fundamental properties of a submersion would be applied to the study of real submanifolds of a complex projective space. In fact, H. B. Lawson [2] has made one step in this direction. His idea is to construct a principal circle bundle $\bar{M}^{2 n}$ over a real hypersurface $M^{2 n-1}$ of $C P^{n}$ in such a way that $\bar{M}^{2 n}$ is a hypersurface of $S^{2 n+1}$ and then to compare the length of the second fundamental tensors of $M^{2 n-1}$ and $\bar{M}^{2 n}$. Thus we can apply theorems on hypersurfaces of $S^{2 n+1}$.

In this paper, using Lawson's method, we prove a theorem which characterizes some remarkable classes of real hypersurfaces of $C P^{n}$. First of all, in $\S 1$, we state a lemma for a hypersurface of a Riemannian manifold of constant curvature for the later use. In $\S 2$, we recall fundamental formulas of a submersion which are obtained in [4], [7] and those established between the second fundamental tensors of $M$ and $\bar{M}$. In $\S 3$, we give some identities which are valid in a real hypersurface of $C P^{n}$. After these preparations, we show, in $\S 4$, a geometric meaning of the commutativity of the second fundamental tensor of $M$ in $C P^{n}$ and a fundamental tensor of the submersion $\pi: \bar{M} \rightarrow M$.

1. Hypersurfaces of a Riemannian manifold of constant curvature. Let $\widetilde{M}$ be an $(m+1)$-dimensional Riemannian manifold with a Riemannian metric $\bar{G}$ and $i: \bar{M} \rightarrow \widetilde{M}$ be an isometric immersion of an $m$-dimensional differentiable

Received by the editors March 25, 1974 and, in revised form, September 9, 1974. AMS (MOS) subject classifications (1970). Primary 53C40, 53C20.

Key words and phrases. Submersion, fundamental tensor of submersion, second fundamental tensor, lift. 
manifold $\bar{M}$ into $\widetilde{M}$. The Riemannian metric $\bar{g}$ of $\bar{M}$ is naturally induced from $\bar{G}$ in such a way that $\bar{g}(\bar{X}, \bar{Y})=\bar{G}(i(\bar{X}), i(\bar{Y}))$, where $\bar{X}, \bar{Y}$ are vector fields on $\bar{M}$ and we denote by the same letter $i$ the differential of the immersion. For an arbitrary point $\bar{x} \in \bar{M}$, we choose a unit normal vector and extend it to a field $\bar{N}$. The Riemannian connections $\bar{D}$ in $\widetilde{M}$ and $\bar{\nabla}$ in $\bar{M}$ are related by the following formulas:

$$
\begin{aligned}
D_{i(\bar{X})} i(\bar{Y}) & =i\left(\bar{\nabla}_{\bar{X}} \bar{Y}\right)+\bar{g}(\overline{H X}, \bar{Y}) \bar{N}, \\
\bar{D}_{i(\bar{X})} \bar{N} & =-i(\overline{H X}),
\end{aligned}
$$

where $\bar{H}$ is the second fundamental tensor of $\bar{M}$ in $\widetilde{M}$.

The mean curvature $\bar{\mu}$ of $\bar{M}$ in $\widetilde{M}$ is defined by

$$
m \bar{\mu}=\operatorname{trace} \bar{H} \text {. }
$$

Let $\widetilde{R}$ and $\bar{R}$ be curvature tensors of $\widetilde{M}$ and of $\bar{M}$ respectively, then we have the following Gauss and Mainardi-Codazzi equations:

$$
\begin{aligned}
\bar{G}(\widetilde{R}(i(\bar{X}), i(\bar{Y})) i(\bar{Z}), i(\bar{W}))= & \bar{g}(R(\bar{X}, \bar{Y}) \bar{Z}, \bar{W})-\bar{g}(\overline{H Y}, \bar{Z}) \bar{g}(\overline{H X}, \bar{W}) \\
& +\bar{g}(\overline{H X}, \bar{Z}) \bar{g}(\overline{H Y}, \bar{W}), \\
\bar{G}(\widetilde{R}(i(\bar{X}), i(\bar{Y})) i(\bar{Z}), \bar{N})= & \bar{g}\left(\left(\bar{\nabla}_{\bar{X}} \bar{H}\right) \bar{Y}, \bar{Z}\right)-\bar{g}\left(\left(\bar{\nabla}_{\bar{Y}} \bar{H}\right) \bar{X}, \bar{Z}\right),
\end{aligned}
$$

where $\bar{X}, \bar{Y}, \bar{Z}$ and $\bar{W}$ are vector fields on $\bar{M}$.

If the ambient manifold is of constant curvature $k$, the curvature tensor $\widetilde{R}$ has the form

$$
\widetilde{R}(\widetilde{X}, \widetilde{Y}) \widetilde{Z}=k\{\bar{G}(\widetilde{Y}, \widetilde{Z}) \widetilde{X}-\bar{G}(\widetilde{X}, \widetilde{Z}) \widetilde{Y}\}
$$

for vector fields $\widetilde{X}, \widetilde{Y}$ and $\widetilde{Z}$ on $\widetilde{M}$. Consequently we have

$$
\begin{aligned}
\bar{R}(\bar{X}, \bar{Y}) \bar{Z} & =k\{\bar{g}(\bar{Y}, \bar{Z}) \bar{X}-\bar{g}(\bar{X}, \bar{Z}) \bar{Y}\}+\bar{g}(\overline{H Y}, \bar{Z}) \overline{H X}-\bar{g}(\overline{H X}, \bar{Z}) \overline{H Y} \\
(\bar{\nabla} \bar{X} \bar{H}) \bar{Y} & =(\bar{\nabla} \bar{Y} \bar{H}) \bar{X} .
\end{aligned}
$$

We assume that $\bar{M}$ has constant mean curvature, that is, trace $\bar{H}=$ const.

Let $\left\{\bar{E}_{1}, \ldots, \bar{E}_{m}\right\}$ be an orthonormal basis in $T_{\bar{x}}(\bar{M})$ and extend them to vector fields in a normal neighborhood of $\bar{x}$ by parallel translation along geodesics with respect to the Riemannian connection of $\bar{M}$. Then we have $\bar{\nabla} \bar{E}_{i}=0 \quad(i=$ $1, \ldots, m)$ at $\bar{x}$. Since $\bar{H}$ and $\bar{\nabla}_{\bar{E}_{i}} \bar{H}$ are both symmetric linear transformations on $T(\bar{M})$, we get, by using (1.8)

$$
\begin{aligned}
\bar{g}\left(\sum_{i=1}^{m}\left(\bar{\nabla}_{\bar{E}_{i}} \bar{H}\right) \bar{E}_{i}, \bar{X}\right) & =\sum_{i=1}^{m} \bar{g}\left(\bar{E}_{i},\left(\bar{\nabla}_{\bar{E}_{i}} \bar{H}\right) \bar{X}\right)=\sum_{i=1}^{m} \bar{g}\left(\bar{E}_{i},\left(\bar{\nabla}_{\bar{X}} \bar{H}\right) \bar{E}_{i}\right) \\
& =\operatorname{trace}\left(\bar{\nabla}_{\bar{X}} \bar{H}\right)=\bar{X}(\operatorname{trace} \bar{H})=0,
\end{aligned}
$$


which implies that

$$
\sum_{i=1}^{m}\left(\bar{\nabla}_{\bar{E}_{i}} \bar{H}\right) \bar{E}_{i}=0
$$

Thus we have

$$
\sum_{i=1}^{m}\left(\bar{\nabla}_{\bar{X}}\left(\bar{\nabla}_{\bar{E}_{i}} \bar{H}\right)\right) \bar{E}_{i}=0 \quad \text { at } \bar{x}
$$

Now we prove the

LEMMA 1.1. Let $\bar{M}$ be a hypersurface of a Riemannian manifold of constant curvature $k$. If the second fundamental tensor $\bar{H}$ satisfies for a constant $\alpha$,

$$
\bar{H}^{2} \bar{X}=\alpha \overline{H X}+k \bar{X}, \quad \bar{X} \in \bar{T}(\bar{M})
$$

then we have $\overline{\nabla H}=0$.

Proof. Since $\bar{H}$ is a symmetric operator and (1.7), (1.8) are valid, we have

$$
\begin{aligned}
\left(\bar{\nabla}_{\bar{X}}\left(\bar{\nabla}_{\bar{Y}} \bar{H}\right)-\bar{\nabla}_{\bar{Y}}\left(\bar{\nabla}_{\bar{X}} \bar{H}\right)-\bar{\nabla}\right. & =k \bar{X}, \bar{Y}] \\
= & k\{\bar{g}(\bar{Y}, \bar{Z} \bar{Z}=\bar{R}) \bar{X}-\bar{g}(\bar{X}, \overline{H Z}) \bar{Y}\}+\overline{H Z}-\bar{H}(\overline{H Y}, \overline{H Z}) \overline{H X}-\bar{g}(\overline{H X}, \overline{H Z}) \overline{H Y} \\
& -k\{\bar{g}(\bar{Y}, \bar{Z}) \overline{H X}-\bar{g}(\bar{X}, \bar{Z}) \overline{H Y}\}-\bar{g}(\overline{H Y}, \bar{Z}) \bar{H}^{2} \bar{X}+\bar{g}(\overline{H X}, \bar{Z}) \bar{H}^{2} \bar{Y}=0 .
\end{aligned}
$$

Let $\left\{\bar{E}_{1}, \ldots, \bar{E}_{m}\right\}$ be an orthonormal basis which is chosen as above and $\bar{X}$ be a tangent vector at $\bar{x}$. Extend $\bar{X}$ to a vector field in a normal neighborhood of $\bar{x}$ by parallel translation along geodesics, then $\overline{\nabla X}=0$ at $\bar{x}$. In the last equation we replace $\bar{Y}$ and $\bar{Z}$ by $\bar{E}_{i}$ and sum over $i$. Then we have, from (1.8) and (1.10),

$$
\sum_{i=1}^{m}\left(\bar{\nabla}_{\bar{E}_{i}}\left(\bar{\nabla}_{\bar{X}} \bar{H}\right)\right) \bar{E}_{i}=\sum_{i=1}^{m}\left(\bar{\nabla}_{\bar{E}_{i}}\left(\bar{\nabla}_{\bar{E}_{i}} \bar{H}\right)\right) \bar{X}=0 \text { at } \bar{X},
$$

because from (1.11) we know that $\bar{M}$ has constant mean curvature. Furthermore (1.11) implies that trace $\bar{H}^{2}=\alpha$ trace $\bar{H}+m k=$ const. Differentiating this covariantly, we have

$$
1 / 2 \overline{Y X}\left(\operatorname{trace} \bar{H}^{2}\right)=\operatorname{trace}\left(\bar{\nabla}_{\bar{Y}}\left(\bar{\nabla}_{\bar{X}} \bar{H}\right)\right) \bar{H}+\operatorname{trace}\left(\bar{\nabla}_{\bar{Y}} \bar{H}\right)\left(\bar{\nabla}_{\bar{X}} \bar{H}\right)=0 \text {, }
$$

from which, at $\bar{x}$,

$$
\operatorname{trace}\left(\bar{\nabla}_{\bar{X}} \bar{H}\right)^{2}=-\operatorname{trace}\left(\bar{\nabla}_{\bar{X}}\left(\bar{\nabla}_{\bar{X}} \bar{H}\right)\right) \bar{H}=-\sum_{i=1}^{m} \bar{g}\left(\left(\bar{\nabla}_{\bar{X}}\left(\bar{\nabla}_{\bar{X}} \bar{H}\right)\right) \bar{E}_{i}, \overline{H E}_{i}\right) .
$$

Thus we have

$$
\bar{g}(\overline{\nabla H}, \overline{\nabla H})=\sum_{i=1}^{m} \operatorname{trace}\left(\bar{\nabla}_{\bar{E}_{i}} \bar{H}\right)^{2}=-\sum_{i, j=1}^{m} \bar{g}\left(\left(\bar{\nabla}_{\bar{E}_{j}}\left(\bar{\nabla}_{\bar{E}_{j}} \bar{H}\right)\right) \bar{E}_{i}, \overline{H E}_{i}\right)=0,
$$

because of (1.12). This completes the proof. 
2. Submersion and immersion. Let $\bar{M}$ and $M$ be differentiable manifolds of dimension $n+1$ and $n$ respectively and assume that there exists a differentiable mapping $\pi$ of $\bar{M}$ onto $M$ which has maximum rank, that is, each differential map $\pi_{*}$ of $\pi$ is onto. Hence, for each $x \in M, \pi^{-1}(x)$ is a 1 -dimensional submanifold of $\bar{M}$, which is called the fibre over $x$. We suppose that every fibre is connected. A vector field on $\bar{M}$ is called vertical if it is always tangent to fibres, horizontal if always orthogonal to fibres; we use corresponding terminology for individual vectors. Thus $\bar{X} \in T_{\bar{x}}(\bar{M})$ decomposes as $\bar{X}^{V}+\bar{X}^{H}$, where $\bar{X}^{V}$ and $\bar{X}^{H}$ denote respectively vertical part and horizontal part of $\bar{X}$.

We assume that the mapping $\pi$ is a Riemannian submersion, that is, there are given in $\bar{M}$ a vertical vector field $\bar{V}$ and a Riemannian metric $\bar{g}$ of $\bar{M}$ satisfying the condition that $\bar{V}$ is a unit Killing vector field with respect to the Riemannian metric $\bar{g}$. Then a Riemannian metric $g$ can be defined on $M$ by

$$
g(X, Y)(x)=\bar{g}\left(X^{L}, Y^{L}\right)(\pi(\bar{x})),
$$

where $\bar{x}$ is an arbitrary point of $\bar{M}$ such that $\pi(\bar{x})=x$ and $X^{L}, Y^{L}$ are the lifts of $X, Y \in T_{x}(M)$ respectively. Hence we have

$$
g(X, Y)^{L}=\bar{g}\left(X^{L}, Y^{L}\right) .
$$

The fundamental tensor $F$ of the submersion $\pi$ is a skew-symmetric tensor of type (1.1) on $M$ and is related to covariant differentiation $\bar{\nabla}$ and $\nabla$ in $\bar{M}$ and $M$, respectively, by the following formulas:

$$
\begin{aligned}
\bar{\nabla}_{Y^{L}} X^{L} & =\left(\nabla_{Y} X\right)^{L}+\bar{g}\left(F^{L} Y^{L}, X^{L}\right) \bar{V}=\left(\nabla_{Y} X\right)^{L}+g(F Y, X)^{L} \bar{V}, \\
\bar{\nabla}_{\bar{V}} X^{L} & =\bar{\nabla}_{X^{L}} \bar{V}=-F^{L} X^{L} .
\end{aligned}
$$

Now we consider two Riemannian submersions $\tilde{\pi}: \widetilde{M} \rightarrow M^{\prime}$ and $\pi: \bar{M} \rightarrow M$ with 1-dimensional fibres and suppose that $\bar{M}$ is a hypersurface of $\widetilde{M}$ which respects the submersion $\tilde{\pi}$. That is, suppose that there are immersions $\tilde{i}: \bar{M} \rightarrow \widetilde{M}$ and $i: M \rightarrow M^{\prime}$ such that the diagram

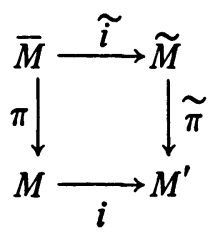

commutes and the immersion $\widetilde{i}$ is a diffeomorphism on the fibres. The commutativity implies that for the unit vertical vector field $\bar{V}$ of $\bar{M}, \tilde{i}(\dot{\bar{V}})$ is also the unit vertical vector field of $\widetilde{M}$ and that for any tangent vector field $X$ to $M, i(X)^{L}=$ $\tilde{i}\left(X^{L}\right)$. Furthermore, for a field of unit normal vector $N$ to $M$ defined in a neigh- 
borhood of $x \in M$, the lift $N^{L}$ is a field of unit normal vectors to $\bar{M}$ defined in a tubular neighborhood of $\bar{x}$, where $\bar{x}$ is an arbitrary point on a fibre over $x$.

We denote by $\bar{D}, \bar{\nabla}, D$ and $\nabla$ the Riemannian connections of $\widetilde{M}, \bar{M}, M^{\prime}$ and $M$ respectively. By means of (1.1), (2.3) and (2.4), we have

$$
\begin{aligned}
\bar{D}_{\tilde{i}\left(X^{L}\right)} \tilde{i}\left(Y^{L}\right) & =\tilde{i}\left(\bar{\nabla}_{X^{L}} Y^{L}\right)+\bar{g}\left(\bar{H} X^{L}, Y^{L}\right) N^{L} \\
& =\widetilde{i}\left(\left(\nabla_{X} Y\right)^{L}+\bar{g}\left(F^{L} X^{L}, Y^{L}\right) \bar{V}\right)+\bar{g}\left(\bar{H} X^{L}, Y^{L}\right) N^{L}, \\
\bar{D}_{\left.\tilde{i}_{(X}\right)} \tilde{i}(\bar{V}) & =\tilde{i}\left(\bar{\nabla}_{X^{L}} \bar{V}\right)+g\left(\overline{H V}, X^{L}\right) N^{L} .
\end{aligned}
$$

Using the above two equations and Gauss equation (1.1) and comparing the vertical parts and horizontal parts, we have

$$
\begin{aligned}
\bar{g}\left(\bar{H} X^{L}, Y^{L}\right) & =g(H X, Y)^{L}, \\
(' F i(X))^{L} & =\widetilde{i}(F X)^{L}-\bar{g}\left(\overline{H V}, X^{L}\right) N^{L},
\end{aligned}
$$

where ' $F$ is the fundamental tensor of the submersion $\tilde{\pi}$. Thus the transforms ' $F i(X)$ and ' $F N$ of $i(X)$ and $N$ by ' $F$ can be written in the form:

$$
\begin{aligned}
\prime F i(X) & =i(F X)+u(X) N, \\
' F N & =-i(U),
\end{aligned}
$$

$u(X)=g(U, X)$. Moreover the following identities are known [1].

$$
\begin{aligned}
\bar{g}\left(\overline{H V}, X^{L}\right) & =-g(U, X)^{L}, \\
\bar{g}(\overline{H V}, \bar{V}) & =0, \\
\operatorname{trace} \bar{H} & =(\operatorname{trace} H)^{L} .
\end{aligned}
$$

LEMMA 2.1. If the second fundamental tensor $\bar{H}$ of the hypersurface $\bar{M}$ is parallel, the second fundamental tensor $H$ of $M$ and the fundamental tensor $F$ of the submersion $\pi$ commutes.

PROOF. Differentiating (2.5) covariantly in the direction of $\bar{V}$ and making use of the fact that $g(H X, Y) \circ \pi$ is invariant along the fibre, we get

$$
\begin{aligned}
\bar{V}(g(H X, Y) \circ \pi) & =\bar{V}\left(\bar{g}\left(\bar{H} X^{L}, Y^{L}\right)\right)=\bar{g}\left(\bar{H}_{\bar{V}} X^{L}, Y^{L}\right)+\bar{g}\left(\bar{H} X^{L}, \bar{\nabla}_{\bar{V}} Y^{L}\right) \\
& =-\bar{g}\left(\bar{H} F^{L} X^{L}, Y^{L}\right)-\bar{g}\left(\bar{H} X^{L}, F^{L} Y^{L}\right) \\
& =-g\left(H F X, Y^{L}+g(F H X, Y)^{L}=0,\right.
\end{aligned}
$$

where we have used (2.4) and the skew-symmetric property of $F$. This completes the proof. 
3. Real hypersurfaces of a complex projective space. Let $S^{n+2}$ be an odddimensional unit sphere in an $(n+3)$-dimensional Euclidean space $E^{n+3}=$ $C^{(n+3) / 2}$ and $\widetilde{J}$ the natural almost complex structure on $C^{(n+3) / 2}$. The image $\widetilde{V}=\widetilde{J} \widetilde{N}$ of the outward unit normal vector $\tilde{N}$ to $S^{n+2}$ by $\widetilde{J}$ defines a tangent vector field on $S^{n+2}$ and the integral curves of $\widetilde{V}$ are great circles $S^{1}$ in $S^{n+2}$ which are the fibres of the standard fibration $\tilde{\pi}$,

$$
S^{1} \rightarrow S^{n+2} \stackrel{\tilde{\pi}}{\longrightarrow} C P^{(n+1) / 2}
$$

onto complex projective space. The usual Riemannian structure on $C P^{(n+1) / 2}$ is characterized by the fact that $\tilde{\pi}$ is a submersion.

Let $M^{n}$ be a real hypersurface of a complex projective space $C P^{(n+1) / 2}$. Then the principal circle bundle $\bar{M}^{n+1}$ over $M^{n}$ is a hypersurface of $S^{n+2}$ and tha natural immersion $\bar{M}^{n+1}$ into $S^{n+2}$ respects the submersion $\tilde{\pi}$. Thus $S^{n+2}$ and $C P^{(n+1) / 2}$ are in the same situations as $\widetilde{M}$ and $M^{\prime}$ respectively, so we continue to use the same notations as those in $\S 2$. In the sequel, we always assume that the hypersurface is connected.

In $S^{n+2}$ we have the family of products $M_{p, q}=S^{p} \times S^{q}$, where $p+q=$ $n+1$. By choosing the spheres to lie in complex subspaces we get fibrations

$$
S^{1} \rightarrow M_{2 p+1,2 q+1} \rightarrow M_{p, q}^{c},
$$

compatible with (3.1), where $p+q=(\dot{n}-1) / 2$. In the special case $p=0$, the hypersurface is a homogeneous, positively curved manifold diffeomorphic to the sphere.

The almost complex structure $J$ of $C P^{(n+1) / 2}$ is nothing but the fundamental tensor of the submersion $\tilde{\pi}$, that is,

$$
J^{L} \widetilde{X}=-\bar{D}_{\widetilde{X}} \widetilde{V}, \quad \widetilde{X} \in T\left(S^{n+2}\right) .
$$

From the discussions of $\S 2$, the transform $J i(X)$ of $i(X)$ by $J$, can be put

$$
J i(X)=i(F X)+g(U, X) N
$$

and we know that $F, U$ and $g$ define the induced almost contact metric structure on $M$. Hence we have, for any $X \in T(M)$,

$$
\begin{aligned}
F^{2} X & =-X+g(U, X) U, \\
g(U, U) & =1, \\
F U & =0 .
\end{aligned}
$$

Differentiating (3.3) covariantly and making use of the fact that the almost complex structure $J$ of $C P^{(n+1) / 2}$ is covariant constant, we have easily 


$$
\begin{aligned}
\left(\nabla_{Y} F\right) X & =u(X) H Y-g(H X, Y) U, \\
\nabla_{Y} U & =F H Y .
\end{aligned}
$$

LEMMA 3.1. $\bar{g}(\overline{H V}, \overline{H V})=1$.

Proof. Let $\bar{x}$ be an arbitrary point of $M$ and $\left\{E_{1}, \cdots, E_{n}\right\}$ be an orthonormal basis at $T_{\pi(\bar{x})}(M)$. We choose an orthonormal basis $\left\{\bar{E}_{1}, \ldots, \bar{E}_{n+1}\right\}$ at $T_{\bar{x}}(\bar{M})$ in such a way that $\bar{E}_{i}=E_{i}^{L}(i=1,2, \ldots, n)$ and $\bar{E}_{n+1}=\bar{V}$. Then, we have

$$
\begin{aligned}
\bar{g}(\overline{H V}, \overline{H V}) & =\sum_{\alpha=1}^{n+1} \bar{g}\left(\overline{H V}, \bar{E}_{\alpha}\right) \bar{g}\left(\overline{H V}, \bar{E}_{\alpha}\right)=\sum_{i=1}^{n} \bar{g}\left(\overline{H V}, E_{i}^{L}\right) \bar{g}\left(\overline{H V}, E_{i}^{L}\right) \\
& =\sum_{i=1}^{n} g\left(U, E_{i}\right) g\left(U, E_{i}\right)=g(U, U)=1,
\end{aligned}
$$

because of (2.9), (2.10) and (3.5).

4. Real hypersurface satisfying a certain commutative condition. In the following we assume that a real hypersurface $M^{n}$ of a complex projective space $C P^{(n+1) / 2}$ satisfies the commutative condition

$$
F H=H F \text {. }
$$

By virtue of Lemma 2.1 if, as a hypersurface of $S^{n+2}$, the principal circle bundle $\bar{M}^{n+1}$ over $M^{n}$ has the parallel second fundamental tensor, then $M$ satisfies (4.1) and $M_{p, q}^{c}$ is an example. In this section we discuss the converse problem, that is, we want to prove that $M_{p, q}^{c}$ is the only hypersurface of $C P^{(n+1) / 2}$ which satisfies (4.1).

We recall the structure equations of a hypersurface of a complex projective space $C P^{(n+1) / 2}$ of the maximal sectional curvature 4:

$$
\begin{gathered}
R(X, Y) Z=g(Y, Z) X-g(X, Z) Y+g(F Y, Z) F X-g(F X, Z) F Y \\
-2 g(F X, Y) F Z+g(H Y, Z) H X-g(H X, Z) H Y, \\
\left(\nabla_{X} H\right) Y-\left(\nabla_{Y} H\right) X=g(U, X) F Y-g(U, Y) F X-2 g(F X, Y) U,
\end{gathered}
$$

where $R$ denotes the curvature tensor of the hypersurface. So we have

$$
g\left(\left(\nabla_{X} H\right) Y, U\right)-g\left(\left(\nabla_{Y} H\right) X, U\right)=-2 g(F X, Y),
$$

because of (3.5) and (3.6). From (4.1) we easily see that $U$ is an eigenvector of $H$, that is,

$$
H U=\alpha U, \quad \alpha=g(H U, U)
$$


Differentiating (4.5) covariantly and making use of (3.8) and (4.1), we have

$$
g\left(\left(\nabla_{X} H\right) Y, U\right)+g\left(H^{2} F X, Y\right)=(X \alpha) g(U, Y)+\alpha g(H F X, Y) .
$$

Forming a similar equation by interchanging $X$ and $Y$ in the last equation and using (4.4), we get

(4.6) $-2 g(F X, Y)+2 g\left(H^{2} F X, Y\right)=(X \alpha) g(U, Y)-(Y \alpha) g(U, X)+2 \alpha g(H F X, Y)$.

In (4.6) if we replace $X$ by $U$, we obtain $Y \alpha=(U \alpha) g(U, Y)$ and substituting this into (4.6) yields $F H^{2} X-\alpha F H X-F X=0$. Transforming this by $F$ and making use of (3.4), we have

$$
H^{2} X-\alpha H X-X+g(U, X) U=0 .
$$

We prove the

LEMMA 4.1. If a hypersurface $M^{n}$ of $C P^{(n+1) / 2}$ satisfies (4.1), the eigenvalue $\alpha$ is constant.

Proof. From the above discussions we have grad $\alpha=\beta U, \beta=U \alpha$. Differentiating this covariantly, we get $\nabla_{X} \operatorname{grad} \alpha=(X \beta) U+\beta F H X$, from which

$$
(Y \beta) g(U, X)-(X \beta) g(U, Y)=2 \beta g(F H X, Y),
$$

because of the fact that $g\left(\nabla_{X} \operatorname{grad} \alpha, Y\right)=g\left(\nabla_{Y} \operatorname{grad} \alpha, X\right)$.

Replacing $X$ by $U$ and making use of (3.5), (3.6), we get $Y \beta=(U \beta) g(U, Y)$. Substituting this into (4.8), we get $\beta g(F H X, Y)=0$. Now let $x$ be a point of $M^{n}$ where $\beta(x) \neq 0$. Then the last equation shows that $F H=0$ at $x$. Hence, from (4.6), $F X=0$. But $F$ has the maximal rank; this is a contradiction. Thus we know that at every point of $M^{n}, \beta=0$. Hence $\alpha$ is constant.

LEMMA 4.2. If the second fundamental tensor $H$ of the hypersurface $M^{n}$ in $C P^{(n+1) / 2}$ satisfies (4.7), the second fundamental tensor $\bar{H}$ of $\bar{M}^{n+1}$ in $S^{n+2}$ satisfies

$$
\bar{H}^{2} \bar{X}=\alpha \overline{H X}+\bar{X},
$$

for any $\bar{X} \in T\left(M^{n+1}\right)$.

Proof. Let $X$ be a tangent vector of $M^{n}$ and first compute $\bar{H}^{2} X^{L}-$ $\alpha \bar{H} X^{L}-X^{L}$ at $\bar{X} \in \bar{M}^{n+1}$. Since any tangent vector $\bar{Y}$ of $\bar{M}^{n+1}$ can be written in the form $\bar{Y}=\bar{Y}^{H}+\bar{Y}^{V}=Y^{L}+\bar{g}(\bar{Y}, \bar{V}) \bar{V}$, at $\bar{x}$, where $Y$ is a tangent vector of $M^{n}$ at $\pi(\bar{x})$, we have

$$
\begin{aligned}
\bar{g}\left(\bar{H}^{2} X^{L}-\alpha \bar{H} X^{L}-X^{L}, \bar{Y}\right)= & \bar{g}\left(\bar{H}^{2} X^{L}-\alpha \bar{H} X^{L}-X^{L}, Y^{L}\right) \\
& +\bar{g}\left(\bar{H}^{2} X^{L}-\alpha \bar{H} X^{L}, \bar{V}\right) \bar{g}(\bar{Y}, \bar{V}) .
\end{aligned}
$$


Since (4.5) implies that $g(H X, U)=\alpha g(U, X)$, it follows from (2.9) that $\bar{g}\left(\bar{H}(H X)^{L}, \bar{V}\right)=-\alpha g(U, X)^{L}$.

On the other hand, (2.5) and the relation $g(H X, Y)^{L}=\bar{g}\left((H X)^{L}, Y^{L}\right)$ show that

$$
\bar{H} X^{L}=(H X)^{L}+\bar{g}\left(\bar{H} X^{L}, \bar{V}\right) \bar{V}=(H X)^{L}-g(X, U)^{L} \bar{V} .
$$

Hence

$$
\bar{H}^{2} X^{L}=\left(H^{2} X\right)^{L}-\alpha g(X, U)^{L} \bar{V}-g(X, U)^{L} \overline{H V}
$$

Thus we have

$$
\bar{H}^{2} X^{L}-\alpha \bar{H} X^{L}-X^{L}=\left(H^{2} X-\alpha H X-X\right)^{L}-g(X, U)^{L} \overline{H V},
$$

and consequently

$$
\begin{aligned}
\bar{g}\left(\bar{H}^{2} X^{L}-\alpha \bar{H} X^{L}-X^{L}, \bar{Y}\right) & \\
= & g\left(H^{2} X-\alpha H X-X+g(X, U) U, Y\right)^{L}=0,
\end{aligned}
$$

because of (2.10) and (4.7).

Next we consider $\bar{H}^{2} \bar{V}-\alpha \overline{H V}-\bar{V}$. For any $\bar{Y} \in T_{\bar{x}}\left(\bar{M}^{n+1}\right)$, we get

$$
\begin{aligned}
\bar{g}\left(\bar{H}^{2} V-\alpha \overline{H V}-\bar{V}, \bar{Y}\right) & =\bar{g}\left(\bar{H}^{2} \bar{V}-\alpha \overline{H V}-\bar{V}, Y^{L}+\bar{g}(\bar{V}, \bar{Y}) \bar{V}\right) \\
& =\bar{g}\left(\bar{H}^{2} \bar{V}, Y^{L}\right)-\alpha \bar{g}\left(\overline{H V}, Y^{L}\right),
\end{aligned}
$$

because of (2.10) and Lemma 3.1.

Making use of (4.12), we have

$$
\bar{g}\left(\bar{H}^{2} \bar{V}-\alpha \overline{H V}-\bar{V}, \bar{Y}\right)=-\alpha g(U, Y)^{L}+\alpha g(U, Y)^{L}=0 .
$$

Combining (4.14) and (4.15), we have (4.9). This completes the proof.

As a consequence of Lemmas 1.1, 2.1 and 4.2, we have

THEOREM 4.3. Let $M^{n}$ be a hypersurface of a complex projective space $C P^{(n+1) / 2}$ and $\pi: \bar{M}^{n+1} \rightarrow M^{n}$ the submersion which is compatible with the fibration $S^{1} \rightarrow S^{n+2} \rightarrow C P^{(n+1) / 2}$. In order that the second fundamental tensor $H$ of $M^{n}$ commute with the fundamental tensor $F$ of the submersion $\pi$, it is necessary and sufficient that the second fundamental tensor $\bar{H}$ of $\bar{M}^{n+1}$ is parallel.

From this theorem and theorems in Ryan's papers [5], [6], we have

THEOREM 4.4. $M_{p, q}^{c}$ are the only complete hypersurfaces of a complex projective space in which the second fundamental tensor $H$ commutes with the fundamental tensor $F$ of the submersion $\pi$. 
Since in [3] we proved that the induced almost contact structure of a hypersurface of a Kaehlerian manifold is normal if and only if $H$ commutes with $F$, we have

COROLlARY 4.5. $M_{p, q}^{c}$ is the only normal almost contact hypersurface of a complex projective space.

\section{BIBLIOGRAPHY}

1. S. Ishihara and M. Konishi, Differential geometry of fibred spaces, A Report in Differential Geometry, Tokyo, 1973.

2. H. B. Lawson, Jr., Rigidity theorems in rank-1 symmetric spaces, J. Differential Geometry 4 (1970), 349-357. MR 42 \#2394.

3. M. Okumura, Certain almost contact hypersurfaces in Kaehlerian manifolds of constant holomorphic sectional curvatures, Tôhoku Math. J. (2) 16 (1964), 270-284. MR 31 \#694.

4. B. O'Neill, The fundamental equations of a submersion, Michigan Math. J. 13 (1966), 459-469. MR 34 \#751.

5. P. J. Ryan, Homogeneity and some curvature conditions for hypersurfaces, Tôhoku Math. J. (2) 21 (1969), 363-388. MR 40 \#6458.

6. - Hypersurfaces with parallel Ricci tensor, Osaka J. Math. 8 (1971), 251-259. MR 45 \#5918.

7. K. Yano and S. Ishihara, Fibred spaces with invariant Riemannian metric, Ködai Math. Sem. Rep. 19 (1967), 317-360. MR 36 \#7075.

DEPARTMENT OF MATHEMATICS, SAITAMA UNIVERSITY, 255 SHIMO OKUBO, URAWA CITY, JAPAN 